\title{
Identification of heat source fields from infra-red thermography: Determination of 'self-heating' in a dual-phase steel by using a dog bone sample
}

Cédric Doudard, ${ }^{\mathrm{a}, 1}$ Sylvain CAlloch ${ }^{\mathrm{a}}$ François Hild ${ }^{\mathrm{b}}$ and Stéphane Roux ${ }^{\mathrm{b}}$

${ }^{a}$ Laboratoire Brestois de Mécanique et des Systèmes (EA 4325) ENSIETA / Université de Brest / ENIB 2 rue François Verny, F-29806 Brest Cedex 9, France. E-mail: Cedric.Doudard@ensieta.fr, Sylvain.Calloch@ensieta.fr

\author{
${ }^{\mathrm{b}} L M T-C a c h a n$
}

ENS de Cachan / CNRS-UMR 8535 / Université Paris 6 / PRES UniverSud Paris 61 avenue du Président Wilson, F-94235 Cachan Cedex, France. E-mail: Francois.Hild@lmt.ens-cachan.fr, Stephane.Roux@lmt.ens-cachan.fr

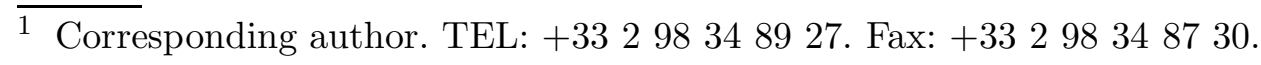




\title{
Identification of heat source fields from infra-red thermography: Determination of 'self-heating' in a dual-phase steel by using a dog bone sample
}

\author{
by \\ C. Doudard, S. Calloch, F. Hild and S. Roux
}

\begin{abstract}
:
From infra-red thermography, a quantitative analysis of heat dissipation sources is proposed via the thermomechanical modeling of a fatigue test on a specimen with a varying cross-section. A new procedure is introduced to achieve this goal, and its application to an experimental case of self-heating at a single load level is shown to provide complete identification of a probabilistic model of micro-plasticity.
\end{abstract}

Key words: thermography, source identification, fatigue, self-heating. 


\section{Introduction}

The measurement and analysis of temperature fields during mechanical tests are often used (i) to analyze a physical phenomenon (e.g., Portevin-Le Châtelier (PLC) bands in aluminum alloys [1, 2], phase transformations in shape memory alloys [3]), (ii) to validate hypotheses (e.g., self-heating localized in a structure to correlate with the region of fatigue initiation [4]), (iii) to identify macroscopic parameters (e.g., velocity of PLC bands $[1,2]$, fatigue properties [5-12], the scatter of fatigue limits [13], multiaxial fatigue criteria $[14,15])$, (iv) or to validate the thermodynamic framework of a macroscopic model [16]. As temperature variation is not totally intrinsic to the material behavior (it depends on the diffusion properties of the materials but also on thermal boundary conditions), the development of constitutive equations in a thermodynamic framework $[17,18]$ requires the determination of the heat source field accompanying these phenomena (e.g., PLC bands, fatigue properties). The heat source field is identified by analyzing temperature fields obtained from infrared images. In this paper, a new method is proposed to carry out this identification.

The reconstruction of heat source maps from the measurements of $2 \mathrm{D}$ temperature fields is performed by solving the heat conduction equation. Many different approaches are available to handle this inverse problem (reconstruction of heat source fields). Due to the ill-posed nature of such problems, an introduction to regularizations and hypotheses about the heat source distribution is required. In the present paper, an analysis of the intrinsic dissipation during cyclic loadings is proposed. Several authors have worked on an estimation of high cycle fatigue properties based upon self-heating measurements 
[10]. The proposed test, hereafter called 'self-heating test', consists in observing thermal effects during cyclic loadings and is performed on a specimen with a constant cross-section. A change of the steady-state temperature occurs beyond a given stress amplitude that is close to the fatigue limit. A model [10] allows us, on the one hand, to describe thermal effects and, on the other hand, to relate the thermal effects to the fatigue properties. The application of this approach to a structure is possible but is not easily interpretable. For the classic test, an analysis of the mean temperature of the specimen is sufficient whereas an average analysis for a structure is seldom applicable [15] so that a general study of the temperature field is necessary to identify the heat source field.

Different methods have been proposed to solve this problem in the studied case (determination of the intrinsic dissipation during cyclic loadings). Among these, Fourier techniques using spectral solutions of the heat conduction equation [19] or special local least-squares fitting of the thermal signal [20] can be mentioned. The first method uses a spectral basis composed of the eigen functions that are compatible with the boundary conditions. In this paper a similar approach is proposed. It is based on a combination of two bases for the projection of the temperature field. The first one, which corresponds to eigen functions not taking into account the boundary conditions, describes the local variations. The second one describes the boundary conditions at the two ends of the specimen. This analysis is performed for the particular case of a 'dog bone' specimen. A one-dimensional (1D) thermal diffusion approach is sufficient for this study.

The paper is divided into three main sections. In the first, the thermodynamic framework and the local heat conduction equation are introduced. To define a 
1D or a 0D thermal diffusion problem, this equation is averaged. In the second section, the proposed approach is presented and illustrated by the study of a $1 \mathrm{D}$ thermal diffusion problem. The same method can also be used in a 2D study. The third section deals with the validation of the proposed approach. The aim is to identify the self-heating properties of the material by using a dog bone specimen.

\section{Heat conduction equation}

The thermodynamics of irreversible processes is used herein. The thermodynamic equilibrium state of a volume element is defined at each instant $t$ by the current value of a set of state variables, namely, $T$, the temperature, $\boldsymbol{\epsilon}$, the strain tensor, or the elastic strain tensor $\boldsymbol{\epsilon}^{e}=\boldsymbol{\epsilon}-\boldsymbol{\epsilon}^{p}$, with $\boldsymbol{\epsilon}^{p}$ the plastic strain tensor, and $V_{k}$ internal variables. Internal variables are chosen in accordance with the physical mechanisms within the studied material. Then, the first and second principles of thermodynamics must be checked for any evolution.

\subsection{Local form}

The local heat conduction equation is deduced from the local form of energy conservation

$$
\rho c \dot{T}+\operatorname{div}(\boldsymbol{q})=\Delta+r+\rho T \frac{\partial^{2} \Psi}{\partial V_{k} \partial T} \cdot \dot{V}_{k}+\rho T \frac{\partial^{2} \Psi}{\partial \boldsymbol{\epsilon}^{e} \partial T}: \dot{\boldsymbol{\epsilon}}^{e}=s_{t}
$$

where $\Psi$ denotes the specific Helmholtz free energy, $c=-T \partial^{2} \Psi / \partial T^{2}$ the specific heat capacity, $\rho$ the mass density, $\boldsymbol{q}$ the heat flux vector, and $s_{t}$ the heat source that is broken down into three parts: 
- intrinsic dissipation $\Delta=\boldsymbol{\Sigma}: \dot{\boldsymbol{\epsilon}}^{p}-A_{k} \cdot \dot{V}_{k} \geq 0$

with $\boldsymbol{\Sigma}=\rho \frac{\partial \Psi}{\partial \boldsymbol{\epsilon}^{e}}$ and $A_{k}=\rho \frac{\partial \Psi}{\partial V_{k}}$;

- external heat supply or density loss $r$;

- thermodynamic coupling source $\rho T \frac{\partial^{2} \Psi}{\partial V_{k} \partial T} \dot{V}_{k}+\rho T \frac{\partial^{2} \Psi}{\partial \boldsymbol{\epsilon}^{e} \partial T}: \dot{\boldsymbol{\epsilon}}^{e}$.

The heat conduction equation (1) allows us to relate the absorption heat capacity $(\rho c \dot{T})$ to the losses by conduction $(\operatorname{div}(\boldsymbol{q}))$ and the heat sources.

\subsection{Application to the self-heating test}

In the case of a self heating test (i.e., the analysis of temperature fields during cyclic mechanical loadings), small temperature variations are observed. Consequently, it is assumed that [19]:

- $c$ is independent of the internal state;

- the convection term included in the material time derivation is neglected (i.e., $d T / d t=\partial T / \partial t)$;

- the external heat supply is time-independent;

- the thermodynamic coupling source is reduced to the thermoelastic contribution (the other contributions are neglected).

The heat source evaluation is an ill-posed problem that is impossible to solve without any information on the heat source distribution. During a classic self-heating test (using a specimen with a constant cross-section) the stress is uniform at a mesoscopic scale, so that the mean heat source is uniform throughout the gauge volume. To identify the mean heat source, the inverse problem may be solved by considering the mean temperature throughout the gauge volume (0D thermal diffusion problem [10, 20]). The regularizing effect 
of heat diffusion limits the thermal gradients so that this mean temperature is measured by a simple thermocouple. For a specimen with a varying crosssection, the stress varies throughout the gauge volume so that the mean heat source distribution is no longer uniform at the considered scale. However for the particular case of the specimen shown in Figure 1, stress essentially depends on height $x$. The inverse problem can be solved by considering the mean temperature for each cross-sectional area, $S(x)$, so that the temperature field depends only on $x$ (1D heat diffusion problem).

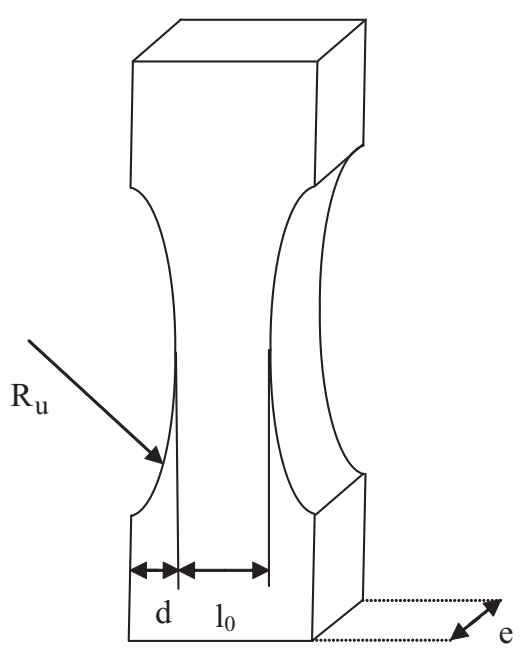

Fig. 1. 'Dog bone' specimen with $R_{u}=80 \mathrm{~mm}$, and $l_{0}=10 \mathrm{~mm}$.

To model local heat conduction, Fourier's law is chosen

$$
\boldsymbol{q}=-\lambda^{\prime} \operatorname{grad} T
$$

where $\lambda^{\prime}$ denotes the isotropic thermal conductivity. In the case of a 1D thermal diffusion problem the conduction losses can be deduced from an analysis of mean losses for a volume element $S(x) d x$

$$
\frac{1}{S(x) d x} \int \operatorname{div}(\boldsymbol{q}) d V=\phi_{\text {long }}+\phi_{\text {lat }}
$$


where $\phi_{\text {long }}$ denotes the conduction losses for height $x$

$$
\phi_{\text {long }}=-\frac{\lambda^{\prime}}{S(x)} \frac{\partial}{\partial x}\left[\frac{\partial \theta}{\partial x}(x, t) S(x)\right]
$$

and $\phi_{\text {lat }}$ denotes the heat exchange with the surrounding environment $[19,21]$

$$
\phi_{l a t}=\left[\frac{2 h_{l}(e+l)}{e l}\right] \theta=\frac{\rho c}{\tau_{e q}^{\prime}} \theta
$$

with $\theta(x, t)=\int_{S} \triangle T d S, h_{l}$ the heat exchange parameter between the specimen and the surrounding air, and $\tau_{e q}^{\prime}$ a characteristic time. Thickness, width and length are denoted by $e, l$ and $L$, respectively.

The longitudinal boundary condition is written as

$$
-\lambda^{\prime} \frac{\partial \theta}{\partial x}( \pm L / 2, t)= \pm h_{2} \theta( \pm L / 2, t)
$$

where $h_{2}$ is the heat-exchange parameter between the specimen and the grips of the testing machine. The characteristic length of the longitudinal losses is therefore defined by $\lambda_{2}^{-1}=\lambda^{\prime} / h_{2}$.

The heat conduction equation is written as a $1 \mathrm{D}$ thermal diffusion problem

$$
\rho c \dot{\theta}(x, t)+\frac{\rho c \theta(x, t)}{\tau_{e q}^{\prime}}-\lambda^{\prime} \frac{\partial^{2} \theta(x, t)}{\partial x^{2}}-\lambda^{\prime} \frac{\partial \theta(x, t)}{\partial x} \frac{\partial S(x)}{\partial x} \frac{1}{S(x)}=S_{t}(x, t),
$$

where $S_{t}(x, t)=\int_{S} s_{t} d S$, with the following boundary condition

$$
-\frac{\partial \theta}{\partial x}( \pm L / 2, t)= \pm \lambda_{2} \theta( \pm L / 2, t)
$$

The heat exchanges with the surrounding air are low compared with the longitudinal losses. Consequently, the second term in the heat conduction equation is neglected. 
In the case of a 0D thermal diffusion problem, a similar analysis of heat losses by conduction allows us to define a characteristic time, $\tau_{e q}$, given by

$$
\frac{1}{\tau_{e q}}=\frac{2 h_{l}(e+l)}{e l}+\frac{2 h_{M}}{L}
$$

where $h_{M}$ is the heat exchange parameter between the grips and the specimen. For a classic servo-hydraulic testing machine, $\tau_{e q}$ is equal to several tens of

seconds, and is low compared with $\tau_{e q}^{\prime}$, that is equal to several hundreds of seconds. Lastly, the local heat conduction is written as a 0D thermal diffusion problem

$$
\rho c \dot{\tilde{\theta}}(t)+\frac{\rho c \widetilde{\theta}(t)}{\tau_{e q}}=\widetilde{S_{t}}(t),
$$

where $\widetilde{S}_{t}(t)=\int_{V} s_{t} d V$ and $\tilde{\theta}(t)=\int_{V} \triangle T d V$.

The following section deals with the proposed method to solve a $1 \mathrm{D}$ thermal diffusion problem (Equation (7)), namely, the identification of the heat source distribution.

\section{Proposed method for identification of heat source distribution}

By using the above method, the present section aims at deducing the heat source distribution from infrared images. This method is based on the properties of the Fourier series. As proposed by Chrysochoos and Louche [19], the temperature distribution is expressed in the spectral basis composed of the eigen functions that are compatible with the boundary conditions. Consequently, the eigen frequencies depend on the heat exchange coefficients. It is therefore necessary to estimate these coefficients accurately. In this paper, an alternative method is proposed: an initial spectral basis disregarding boundary conditions is combined with additional components to describe the exchanges. 
To present this method, a specimen with a constant cross-section is considered.

\subsection{Spectral basis}

For the considered basis, the 1D thermal diffusion problem is solved regardless of boundary losses at $x= \pm L / 2$. The heat sources being neglected, the local heat conduction equation becomes

$$
\dot{\theta}(x, t)+\frac{\theta(x, t)}{\tau_{e q}^{\prime}}-\frac{\lambda^{\prime}}{\rho c} \frac{\partial^{2} \theta(x, t)}{\partial x^{2}}=0 .
$$

If $\theta(x, t)=\phi(t) w(x)$, Equation (11) reads

$$
\frac{\rho c}{\lambda^{\prime}} \frac{\dot{\phi}(t)}{\phi(t)}+\frac{\rho c}{\lambda^{\prime} \tau_{e q}^{\prime}}=\frac{1}{w(x)} \frac{\partial^{2} w(x)}{\partial x^{2}}=-\delta .
$$

By choosing $\sqrt{\delta}=w_{i}=2 \pi i / L$, the solution is expressed as

$$
w(x)=\sum_{i} A_{i} \sin \left(w_{i} x\right)+B_{i} \cos \left(w_{i} x\right)
$$

where $A_{i}$ and $B_{i}$ are heat-source-dependent parameters. Consequently the temperature is related to the thermal source by

$$
\begin{aligned}
\theta(x, t)= & \sum_{i}\left[\int_{0}^{t}\left\langle\frac{S_{t}(x, s)}{\rho c}, \cos \left(w_{i} x\right)\right\rangle \exp \left(-\overline{w_{i}^{\prime}}(t-s)\right) d s\right] \cos \left(w_{i} x\right) \\
& +\left[\int_{0}^{t}\left\langle\frac{S_{t}(x, s)}{\rho c}, \sin \left(w_{i} x\right)\right\rangle \exp \left(-\overline{w_{i}^{\prime}}(t-s)\right) d s\right] \sin \left(w_{i} x\right)
\end{aligned}
$$

where $\langle a(x), b(x)\rangle=\int_{-\frac{L}{2}}^{\frac{L}{2}} a(x) b(x) d x$ and $\overline{w_{i}^{\prime}}=w_{i}^{2} \lambda^{\prime} / \rho c+1 / \tau_{e q}^{\prime}$.

With the considered basis and a uniform heat source $S_{t 0}$, the mean steady state temperature variation is given by

$$
\overline{\widetilde{\theta}}=\frac{S_{t 0}}{\rho c} \tau_{e q}^{\prime} \neq \frac{S_{t 0}}{\rho c} \tau_{e q}
$$


where $S_{t 0} \tau_{e q} / \rho c$ denotes the responses obtained with a $0 \mathrm{D}$ approach. This result shows the need to take into account the heat losses due to exchanges with the grips in order to estimate heat diffusion accurately. There are two ways of solving this problem. The first consists in defining eigen functions that are compatible with the boundary conditions. However, it is necessary to evaluate the heat exchange coefficients beforehand. The second consists in completing the initial basis. It is assumed that the boundary losses have a significant influence on the mean response but a weaker influence on local variation.

It is therefore proposed to complete the initial basis by a second degree polynomial. This choice allows us to account for arbitrary heat fluxes at both ends of the specimen $x= \pm L / 2$.

\subsection{Polynomial basis}

It is considered that $\theta(x)=a x^{2}+b x+c$. Leaving aside the losses due to exchanges with the surrounding air because they are lower than these caused by exchanges with the grips, the steady state heat local conduction equation is written as

$$
S t_{0}=-2 a \lambda^{\prime}
$$

The boundary conditions are given by

$$
\begin{aligned}
& -2 a \frac{L}{2}+b=\lambda_{2}\left[a \frac{L^{2}}{4}-b \frac{L}{2}+c\right], \\
& 2 a \frac{L}{2}+b=-\lambda_{2}\left[a \frac{L^{2}}{4}+b \frac{L}{2}+c\right] .
\end{aligned}
$$


These equations allow us to relate the three parameters $a, b$ and $c$ to the uniform heat source and the heat exchange coefficients by

$$
a=-\frac{S t_{0}}{2 \lambda^{\prime}} \quad, \quad b=0 \quad, \quad c=-\left(\frac{a L}{\lambda_{2}}+a \frac{L^{2}}{4}\right) .
$$

In the considered case, the problem is symmetrical. Consequently $b=0$. The proposed polynomial basis allows therefore the description of the 'mean' behavior. In the following section, a solution to the 1D thermal diffusion problem using the complete basis is proposed.

\subsection{Orthogonal basis}

The proposed basis to solve the 1D problem is not orthogonal because of the linear and quadratic polynomial terms. It is therefore proposed to subtract from them their projection over the Fourier series. The first order polynomial, $Q(x)=b x$, can easily be projected over the eigen functions to give

$$
Q_{/ F}=\sum_{k} \frac{-b L(-1)^{k}}{\pi k} \sin \left(\frac{2 \pi k x}{L}\right) .
$$

The same computation can be performed for the second order polynomial $P(x)=a x^{2}$, whose projection can be written

$$
P_{/ F}=\frac{a L^{2}}{12}+\sum_{k} \frac{a L^{2}(-1)^{k}}{\pi^{2} k^{2}} \cos \left(\frac{2 \pi k x}{L}\right) .
$$

Consequently, the following orthogonal basis is chosen

$$
\begin{aligned}
{\left[U_{a}=P-P_{/ F}, U_{b}=Q-Q_{/ F}, U_{0}\right.} & =1 \\
U_{k}=\cos \left(\frac{2 \pi k x}{L}\right), V_{k} & \left.=\sin \left(\frac{2 \pi k x}{L}\right)\right]
\end{aligned}
$$

where $k>0$. The projection of the temperature signal onto the three first terms leads to the identification of $a, b$ and $c . \theta_{a k}$ and $\theta_{b k}$ denote the pro- 
jection onto the other terms. Consequently, the change of the steady-state temperature variation is described by

$$
\theta=a x^{2}+b x+c+\sum_{k>0} \theta_{a k}^{\prime} \cos \left(\frac{2 \pi k x}{L}\right)+\theta_{b k}^{\prime} \sin \left(\frac{2 \pi k x}{L}\right)
$$

with $\theta_{a k}^{\prime}=\theta_{a k}-a L^{2}(-1)^{k} / \pi^{2} k^{2}$ and $\theta_{b k}^{\prime}=\theta_{b k}+b L(-1)^{k} / \pi k$. The heat source distribution is then related to the temperature distribution by

$$
S t_{0}=-2 a \lambda^{\prime} \quad, \quad S t_{a k}=\rho c \bar{w}_{k}^{2} \theta_{a k}^{\prime} \quad, \quad S t_{b k}=\rho c \bar{w}_{k}^{2} \theta_{b k}^{\prime}
$$

with $\bar{w}_{k}=\left(\frac{2 \pi k}{L}\right)^{2} \frac{\lambda^{\prime}}{\rho c}$.

The proposed basis allows us to take into account heat losses due to the exchange with the grips and is easy to implement (i.e., it is not necessary to identify different parameters beforehand). An illustration of the present approach with the analysis of self-heating measurements of a 'dog bone' specimen during cyclic loadings is proposed.

\section{Self-heating properties in a 'dog bone' specimen}

\subsection{Self-heating test}

The identification of high cycle fatigue $(\mathrm{HCF})$ properties from traditional tests is time consuming and very expensive. Alternative experimental methods were developed to identify $\mathrm{HCF}$ characteristics [5, 7, 22-25] more rapidly. Among these, the self-heating test monitors the specimen temperature changes during cyclic loadings (usually a 0D approach is used to analyze tests). It consists in applying successive series of cycles with different increasing stress amplitudes on a specimen with a constant cross-section. For each stress amplitude, the 
change in the temperature variation, $\widetilde{\theta}$ is recorded. The mean temperature becomes stable after several hundred cycles and equals $\overline{\widetilde{\theta}}$ depending on stress level $\Sigma_{0}$ and loading frequency $f_{r}$. Figure 2 shows the self-heating curve for a dual-phase steel (i.e., the change of $\overline{\widetilde{\theta}}$ with the load amplitude). Beyond a given stress level the mean steady-state temperature starts to increase significantly. This regime change corresponds to microplastic activation that induces an intrinsic dissipation.

To describe the thermal effects, a probabilistic approach was put forward. If it is assumed that HCF damage is caused by this microplastic activity, the proposed approach allows the description of the fatigue behavior within the same framework [13]. The dissipated energy density for a loading cycle $D_{\text {cycle }}$ is related to the stress amplitude by

$$
D_{\text {cycle }}=\delta \Sigma_{0}^{m+2}
$$

where $\delta$ is a parameter depending on the material and $m$ the Weibull modulus. The mean temperature variation induced by this dissipation is determined by solving the local heat conduction equation (0D approach)

$$
\rho c \dot{\tilde{\theta}}(t)+\frac{\rho c \tilde{\theta}(t)}{\tau_{e q}}=f_{r} D_{\text {cycle }} .
$$

The mean steady-state temperature is also related to the stress amplitude by

$$
\overline{\widetilde{\theta}}=\frac{f_{r} \delta \tau_{e q}}{\rho c} \Sigma_{0}^{m+2}
$$

Consequently the Weibull modulus can be identified from self-heating measurements [13] (see Figure 2). For the considered material, a value $m=12.5$ is obtained.

In the following section, an analysis of self-heating measurements is proposed 


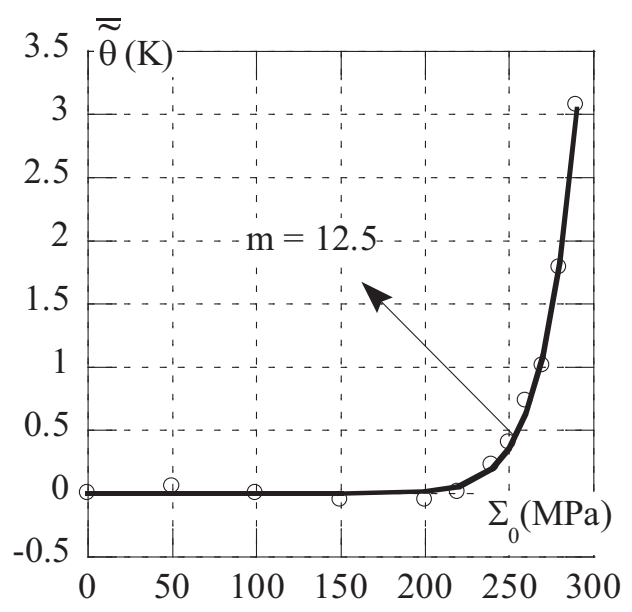

Fig. 2. Self-heating curve of a dual-phase steel. Change of the steady-state mean temperature with the stress amplitude $\left(f_{r}=10 \mathrm{~Hz}\right)$.

for a 'dog bone' specimen for which the 1D approach will be used.

\section{2 'Dog bone' specimen}

For a 'dog bone' specimen shown in Figure 1, the longitudinal stress depends only on the height $x$ and the cross-sectional area is related to $x$ by

$$
S(x)=e l_{0}\left[1+\frac{2 R_{u}}{l_{0}}\left(1-\sqrt{1-\left(\frac{x}{R_{u}}\right)^{2}}\right)\right],
$$

where $x=0$ corresponds to the smaller cross-section. The stress amplitude is also given by

$$
\Sigma_{0}(x)=\frac{\Sigma_{0 M}}{\left[1+\frac{2 R_{u}}{l_{0}}\left(1-\sqrt{1-\left(\frac{x}{R_{u}}\right)^{2}}\right)\right]},
$$

where $\Sigma_{0 M}$ is the largest stress amplitude (i.e., for $x=0$ ).

Consequently, the heat source reads

$$
S_{t}(x)=\delta f_{r} \Sigma_{0}(x)^{m+2}=\delta f_{r}\left(\frac{\Sigma_{0 M}}{\left[1+\frac{2 R_{u}}{l_{0}}\left(1-\sqrt{1-\left(\frac{x}{R_{u}}\right)^{2}}\right)\right]}\right)^{m+2},
$$


The self-heating test with a 'dog bone' specimen consists in applying one series of cycles with a constant stress amplitude. The heat source distribution is obtained from infrared images by solving the local heat conduction equation. The change of the heat source with the stress amplitude is deduced directly. This result can thus be used to identify the Weibull modulus $m$. It is proposed to validate this identification method of the Weibull modulus by comparing the values of $m$ obtained from a 'dog bone' specimen and another value with a constant cross-section. However, it is necessary to develop the above identification method for the case of a specimen with a non-constant cross-section.

\subsection{Application to a varying cross-section}

The local heat conduction equation for a specimen with a non-constant crosssection is given by (1D approach)

$$
\rho c \dot{\theta}(x, t)-\lambda^{\prime} \frac{\partial^{2} \theta(x, t)}{\partial x^{2}}-\lambda^{\prime} \frac{\partial \theta(x, t)}{\partial x} \frac{\partial S(x)}{\partial x} \frac{1}{S(x)}=S_{t}(x, t) .
$$

If the temperature field is expanded as follows

$$
\theta=a x^{2}+b x+c+\sum_{k>0} \theta_{a k}^{\prime} \cos \left(w_{k} x\right)+\theta_{b k}^{\prime} \sin \left(w_{k} x\right)
$$

where $w_{k}=2 \pi k / L$, Equation (31) becomes

$$
\begin{array}{r}
-\lambda^{\prime} 2 a(1+g(x))-\lambda^{\prime} b \frac{h(x)}{L} \\
+\lambda^{\prime}\left[\sum_{k>0} w_{k}^{2} \theta_{a k}^{\prime} \cos \left(w_{k} x\right)+\theta_{b k}^{\prime} \sin \left(w_{k} x\right)\right] \\
+\lambda^{\prime}\left[\sum_{k>0} w_{k} \theta_{a k}^{\prime} \sin \left(w_{k} x\right) \frac{h(x)}{L}-w_{k} \theta_{b k}^{\prime} \cos \left(w_{k} x\right) \frac{h(x)}{L}\right]=S_{t}(x),
\end{array}
$$

with

$$
g(x)=\frac{\partial S(x)}{\partial x} \frac{x}{S(x)} \quad \text { and } \quad h(x)=\frac{\partial S(x)}{\partial x} \frac{L}{S(x)} .
$$


Consequently, the following relationships are obtained

$$
\begin{array}{r}
S t_{0}=-2 a \lambda^{\prime}\left(1+g_{0}\right)-\lambda^{\prime} b \frac{h_{0}}{L}+\lambda^{\prime} \\
{\left[\sum_{k>0} w_{k} \theta_{a k}^{\prime} \frac{h_{b k}}{2 L}-w_{k} \theta_{b k}^{\prime} \frac{h_{a k}}{2 L}\right],} \\
S t_{a k}=\lambda^{\prime}\left(w_{k}^{2} \theta_{a k}^{\prime}-2 a g_{a k}-b \frac{h_{a k}}{L}+\right. \\
\left.\sum_{j>0} \frac{w_{j}}{2 L}\left[\theta_{a j}^{\prime}\left(h_{b j-k}+h_{b j+k}\right)-\theta_{b j}^{\prime}\left(h_{a j+k}+h_{a j-k}\right)\right]\right), \\
S t_{b k}=\lambda^{\prime}\left(w_{k}^{2} \theta_{b k}^{\prime}-2 a g_{b k}-b \frac{h_{b k}}{L}\right. \\
\left.+\sum_{j>0} \frac{w_{j}}{2 L}\left[\theta_{a j}^{\prime}\left(h_{a j-k}-h_{a j+k}\right)-\theta_{b j}^{\prime}\left(h_{b j+k}-h_{b j-k}\right)\right]\right) .
\end{array}
$$

These three relationships allow us to relate the heat source distribution to the temperature distribution.

Despite all the precautions used for data acquisition, temperature measurements are noisy. In the following, it is proposed to determine the relevant harmonic number to achieve a good estimation of the heat source distribution.

\subsection{Relevant harmonic number}

With the considered basis, the larger the harmonic of the eigen basis, the smaller the signal-to-noise ratio (see Equation (14)). Consequently it is necessary to choose the dimension of the eigen basis, $k_{l i m}$. For a general study, it is difficult to give a unique answer. However, in the case considered, the general trend of the heat source distribution is given by Equation (30). It is thus possible to determine the relevant harmonic number to achieve a good estimation of the Weibull modulus $m$ by considering a temperature field without noise. 
This temperature distribution is obtained from FE simulations by considering $\lambda^{\prime}=64 \mathrm{Wm}^{-1} \mathrm{~K}^{-1}, \lambda_{2}=70 \mathrm{~m}^{-1}$ and $m=12$. Figure 3a shows the change of the steady-state temperature variation with $x$ obtained by this method. From the temperature change, the proposed method allows the identification of the heat source distribution for different values of $k_{\text {lim }}$ (relevant number of harmonics), see Figure 3b.
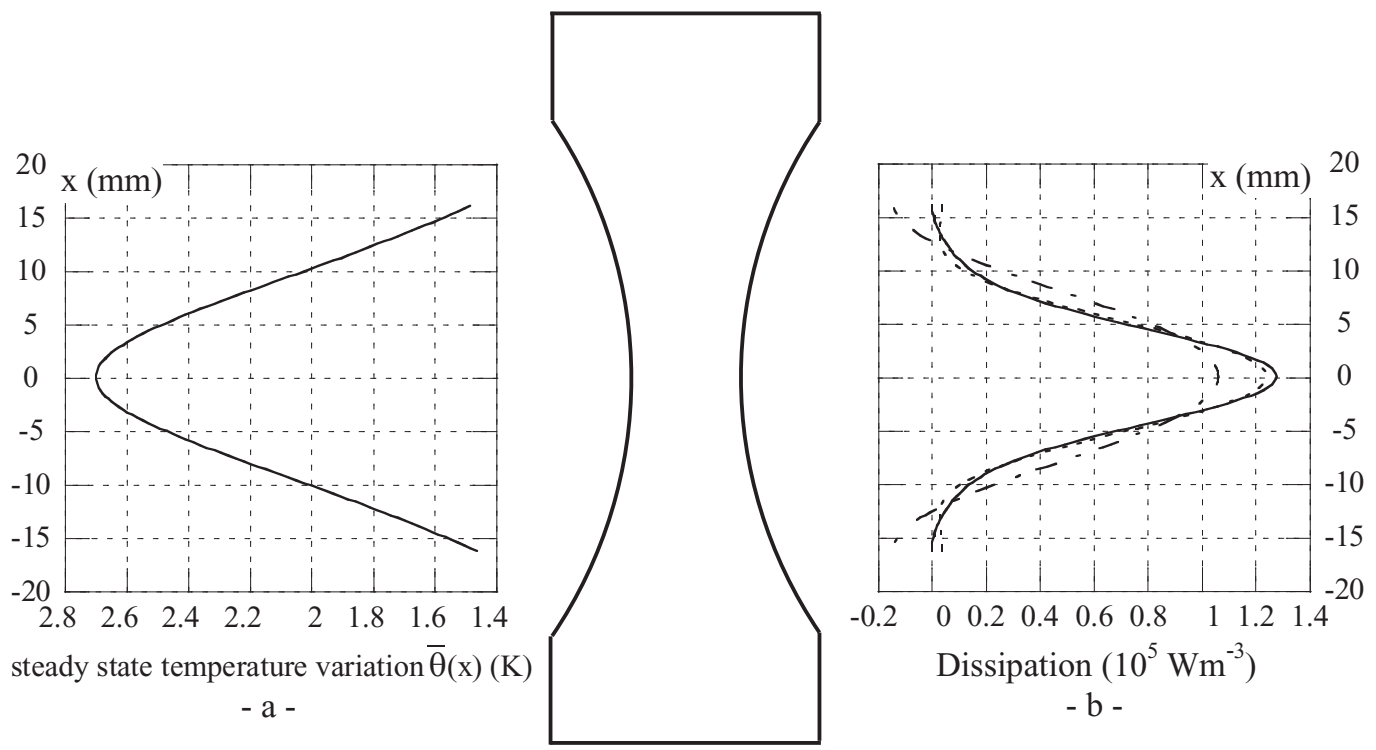

Fig. 3. -a-Change of the steady-state temperature variation with $x\left(f_{r}=10 \mathrm{~Hz}\right.$ and $\lambda^{\prime}=64 \mathrm{Wm}^{-1} \mathrm{~K}^{-1}$ ) obtained from a numerical simulation -b-Identified heat source distribution from temperature changes for different values of $k_{\text {lim }}$. Dotted line: $k_{\text {lim }}=1$, dashed line: $k_{\text {lim }}=2$, solid line: $k_{\text {lim }}=3$.

For each heat source distribution, the value of $m$ is determined (Table 1). Consequently, $k_{\text {lim }}=3$ is considered as sufficient for the following study.

\section{Table 1}

\begin{tabular}{|c|c|c|c|c|}
\hline$k_{\text {lim }}$ & 1 & 2 & 3 & 4 \\
\hline$m$ & 5.3 & 10.5 & 11.9 & 11.9 \\
\hline
\end{tabular}

Identified values of $m$ from the heat source distribution for different values of $k_{\text {lim }}$ (considered number of harmonics). The reference is $m=12$. 


\subsection{Experiment on a dual-phase steel}

A self-heating test is carried out on a servohydraulic testing machine. This load-controlled test has a 320-MPa stress amplitude (when $x=0$ ) and a $10-\mathrm{Hz}$ frequency. The temperature field is monitored using an infrared camera (JADE IRFPA, resolution: $320 \times 356$ pixels, medium wave with a $3-5 \mu m$ spectral range). Figure $4 \mathrm{a}$ shows the change of the steady-state temperature variation with $x$ (average over 25 pixels in the width direction). By using the proposed method, the heat source distribution is identified (Figure 4b).
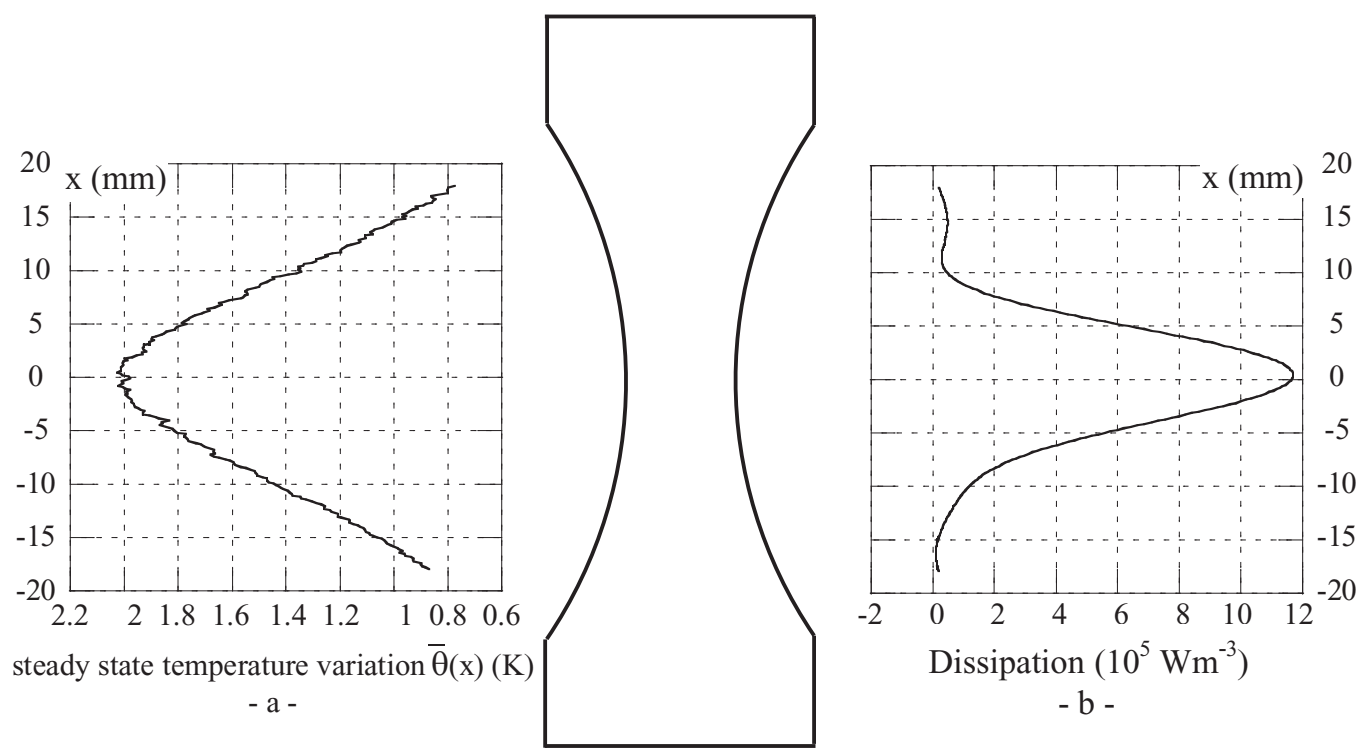

Fig. 4. -a-Change of the steady-state temperature variation with $x$ given by an infrared camera $\left(f_{r}=10 H z\right)$-b-Identified heat source distribution from temperature change with $k_{\text {lim }}=3$ and $\lambda^{\prime}=64 \mathrm{Wm}^{-1} \mathrm{~K}^{-1}$.

Figure 5 shows the self-heating curve of the material considered deduced from the present analysis (i.e., the heat source distribution with a unique stress amplitude). A value of $m=12$ is obtained. This value is in accordance with that obtained from traditional self-heating tests (i.e., $m=12.5$, Figure 2). This result validates, on the one hand, the proposed identification method 
and, on the other hand, the use of a 'dog bone' specimen to identify the value of the Weibull modulus.

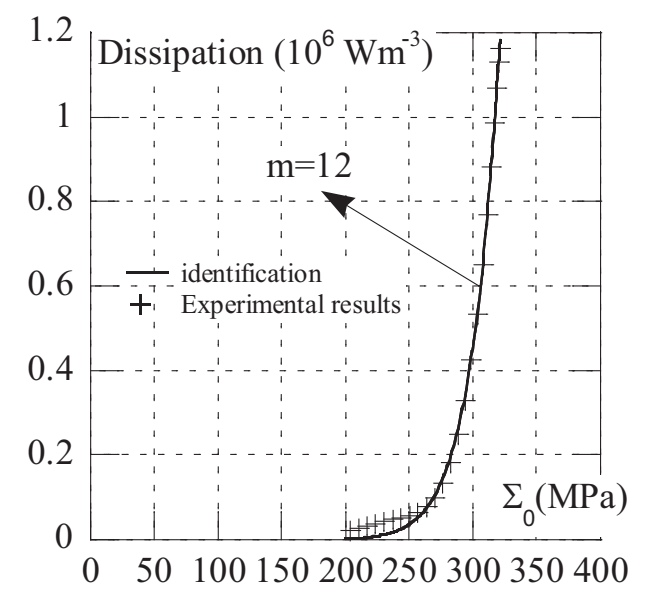

Fig. 5. Self-heating curve of a dual-phase steel (heat source change with the stress amplitude $(\mathrm{MPa}))$. Solid line: identified change $(m=12)$, Symbol + : experimental data.

\section{Conclusion}

The method proposed herein to identify heat source distributions from infrared images is based on the use of a new basis for the projection of the temperature field. It is broken down into two parts. The first one, which corresponds to an eigen basis regardless of the boundary conditions, is used to describe the local variations of the temperature field. The second describes the boundary conditions at the two ends. Three advantages can be underlined. First, this approach is easy to implement. Second, it is not necessary to identify the heat exchange parameters. Third, it is possible to define a region of interest that is independent of the length of the specimen gauge part.

The validation of the proposed approach is performed by analyzing a selfheating test of a 'dog bone' sample made of dual-phase steel. The results with 
this new method are in accordance with those determined from a traditional configuration (i.e., specimen with a constant cross-section). The proposed approach may be used to analyze the self-heating of structures and to identify their fatigue properties. 


\section{References}

[1] N. Ranc, D. Wagner, Some aspects of Portevin-Le Chatelier plastic instabilities investigated by infrared pyrometry, Mater. Sc. Engng. A394:8795, 2005.

[2] H. Louche, P. Vacher, R. Arrieux, Thermal observations associated with the Portevin-Le Chatelier effect in an Al-Mg alloy, Mater. Sc. Engng. A 404:188-196, 2005.

[3] X. Balandraud, A. Chrysochoos, S. Leclercq, R. Peyroux, Effet du couplage thermomécanique sur la propagation d'un front de changement de phase, C.R. Mécanique, 329:621-626, 2001.

[4] E. Charkaluk, A. Bigonnet, A. Constantinescu and K. Dang Van, Fatigue Design of Structures under Thermomechanical Loadings, Fat. Fract. Engng. Mater. Struct., 25:1199-1206, 2002.

[5] M. P. Luong and K. Dang Van, Metal Fatigue Limit Evaluation Using Infrared Thermography, Proceedings of AITA, Capri (Italy), 1994.

[6] J.-C. Krapez, D. Pacou and C. Bertin, Application of lock-in thermography to rapid evaluation of fatigue limit in metals, Proceedings of the $5^{\text {th }}$ AITA, Venezia (Italy), 1999.

[7] G. La Rosa and A. Risitano, Thermographic methodology for rapid determination of the fatigue limit of materials and mechanical components, Int. J. Fatigue, 22:65-73, 2000.

[8] P. K. Liaw, H. Wang, L. Jiang, B. Yang, J. Y. Huang, R. C. Kuo and J. C. Huang, Thermographic detection of fatigue damage of pressure vessel steels at $1000 \mathrm{~Hz}$ and $20 \mathrm{~Hz}$, Scripta Mat., 42:389-395, 2000.

[9] A. E. Morabito, V. Dattoma and U. Galietti, Energy-analysis of fatigue damage by thermographic technique, SPIE, 4710:460-467, 2002. 
[10] C. Doudard, S. Calloch, F. Hild, P. Cugy and A. Galtier, A probabilistic two-scale model for high cycle fatigue life predictions, Fat. Fract. Engng. Mater. Struct., 28:279-288, 2005.

[11] G. Meneghetti, Analysis of the fatigue strength of a stainless steel based on the energy dissipation, Int. J. Fatigue, 29:81-94, 2007.

[12] T. Ummenhofer, J. Medgenberg, On the use of infrared thermography for the analysis of fatigue damage processes in welded joints, Int. J. Fatigue, 31:130-137, 2009.

[13] C. Doudard, S. Calloch, F. Hild, P. Cugy and A. Galtier, Identification of the scatter in high cycle fatigue from temperature measurements, C.R. Mécanique, 332:795-801, 2004.

[14] C. Doudard, M. Poncelet, S. Calloch, C. Boue, F. Hild and A. Galtier, Determination of an HCF criterion by thermal measurements under biaxial cyclic loading, Int. J. Fatigue, 29:748-757, 2007.

[15] M. Poncelet, C. Doudard, S. Calloch, F.Hild, B. Weber and A. Galtier, Prediction of self-heating measurements under proportional and nonproportional multiaxial cyclic loadings, C.R. Mcanique, 335:81-86, 2007.

[16] L. Vincent, On the ability of some cyclic plasticity models to predict the evolution of stored energy in a type 304L stainless steel submitted to high cycle fatigue, Eur. J. Mech. - A/Solids, 27:161-180, 2008.

[17] P. Germain, Q. S. Nguyen and P. Suquet, Continuum Thermodynamics, ASME J. Appl. Mech. 50 (1983) 1010-1020.

[18] J. Lemaitre and J.-L. Chaboche, Mechanics of Solid Materials, (Cambridge University Press, Cambridge (UK), 1990).

[19] A. Chrysochoos and H. Louche, An infrared image processing to analyse the calorific effects accompanying strain localisation, Int. J. Eng. Sc., 38:1759-1788, 2000. 
[20] T. Boulanger, A. Chrysochoos, C. Mabru and A. Galtier, Calorimetric Analysis of Dissipative and Thermoelastic Effects Associated with the Fatigue Behavior of Steels, Int. J. Fatigue, 26:221-229, 2004.

[21] A. B. De Vriendt, La Transmission de la Chaleur, Morin, Québec (Canada), 1987.

[22] C. E. Stromeyer, The determination of Fatigue Limits under Alternating Stress Conditions, Proc. Roy. Soc. (A), 90:411-425, 1914.

[23] H. F. Moore and J. B. Kommers, Fatigue of Metals Under Repeated Stress, Chem. Met. Eng., 25:1141-1144, 1921.

[24] E. Lehr, Die Dauerfestigkeit, ihre Bedeutung für die Praxis und ihre kurzfristige Ermittlung mittels neuartiger Prüfmaschinen, Glasers Ann. Gew., 99:109-114, 1926.

[25] G. Welter, Bending and tension-compression fatigue testing, Wiad. Inst. Metal., 4:30-39, 1937. 